\title{
Neuroprotective Roles of the Adenosine A3 Receptor Agonist AST-004 in Mouse Model of Traumatic Brain Injury
}

Eda Bozdemir

UT Health San Antonio: The University of Texas Health Science Center at San Antonio Fabio A Vigil

UT Health San Antonio: The University of Texas Health Science Center at San Antonio Sang H Chun

UT Health San Antonio: The University of Texas Health Science Center at San Antonio Liliana Espinoza

UT Health San Antonio: The University of Texas Health Science Center at San Antonio

Vladislav Bugay

UT Health San Antonio: The University of Texas Health Science Center at San Antonio

\section{Sarah M Khoury}

UT Health San Antonio: The University of Texas Health Science Center at San Antonio Deborah M Holstein

UT Health San Antonio: The University of Texas Health Science Center at San Antonio Aiola Stoja

UT Health San Antonio: The University of Texas Health Science Center at San Antonio Damian Lozano

UT Health San Antonio: The University of Texas Health Science Center at San Antonio Ceyda Tunca

UT Health San Antonio: The University of Texas Health Science Center at San Antonio Shane M Sprague

UT Health San Antonio: The University of Texas Health Science Center at San Antonio Jose E Cavazos

UT Health San Antonio: The University of Texas Health Science Center at San Antonio

Robert Brenner

UT Health San Antonio: The University of Texas Health Science Center at San Antonio Theodore E Liston

Astrocyte Pharmaceuticals Inc

\section{Mark S Shapiro}

UT Health San Antonio: The University of Texas Health Science Center at San Antonio James Donald Lechleiter ( $\square$ lechleiter@uthscsa.edu ) 
UT Health San Antonio https://orcid.org/0000-0001-9000-0722

\section{Research}

Keywords: ADORA3, concussion treatment, mitochondrial metabolism, ATP, astrocytes

Posted Date: January 18th, 2021

DOI: https://doi.org/10.21203/rs.3.rs-145867/v1

License: (c) (i) This work is licensed under a Creative Commons Attribution 4.0 International License. Read Full License

Version of Record: A version of this preprint was published at Neurotherapeutics on October 4th, 2021. See the published version at https://doi.org/10.1007/s13311-021-01113-7. 


\section{Abstract}

Background: Traumatic brain injury (TBI) remains one of the greatest public health concerns with increasing morbidity and mortality rates worldwide. Our group reported stimulation of astrocyte mitochondrial metabolism by $\mathrm{P} 2 \mathrm{Y} 1$ receptor agonists significantly reduced cerebral edema and reactive gliosis in a TBI model. Subsequent data on the pharmacokinetics (PK) and rapid metabolism of these compounds suggested neuroprotection was likely mediated by a metabolite, AST-004, which binding data indicated was an adenosine $\mathrm{A} 3$ receptor (A3R) agonist.

Methods: The neuroprotective efficacy of AST-004 was tested in a controlled closed cortical injury (CCCI) model of TBI in mice.

Results: Twenty-four (24) hours post-injury, mice subjected to CCCI and treated with AST-004 $(0.22 \mathrm{mg} / \mathrm{kg})$ exhibited significantly less secondary brain injury. These effects were quantified with less cell death (PSVue794 fluorescence) and loss of blood brain barrier breakdown (Evans Blue extravasation assay), compared to vehicle treated TBI mice. TBI treated mice also exhibited significantly reduced neuroinflammatory markers, glial-fibrillary acidic protein (GFAP, astrogliosis) and ionized $\mathrm{Ca} 2+$ binding adaptor molecule 1 (Iba1, microgliosis), both at the mRNA (gRT-PCR) and protein (western blot and immunofluorescence) levels, respectively. Four (4) weeks post-injury, AST-004 treated TBI mice presented significantly reduced impairment of long-term memory. Spatial memory was assessed with a contextual fear conditioning behavior assay (freezing behavior after shock). Finally, AST-004 treatments were found to increase in vivo ATP production in astrocytes (gfap-targeted luciferase activity), consistent with the proposed mechanism of action.

Conclusions: These data reveal AST-004 as a novel A3R agonist that increases astrocyte energy production and enhances their neuroprotective efficacy after brain injury.

\section{Full Text}

This preprint is available for download as a PDF.

\section{Figures}



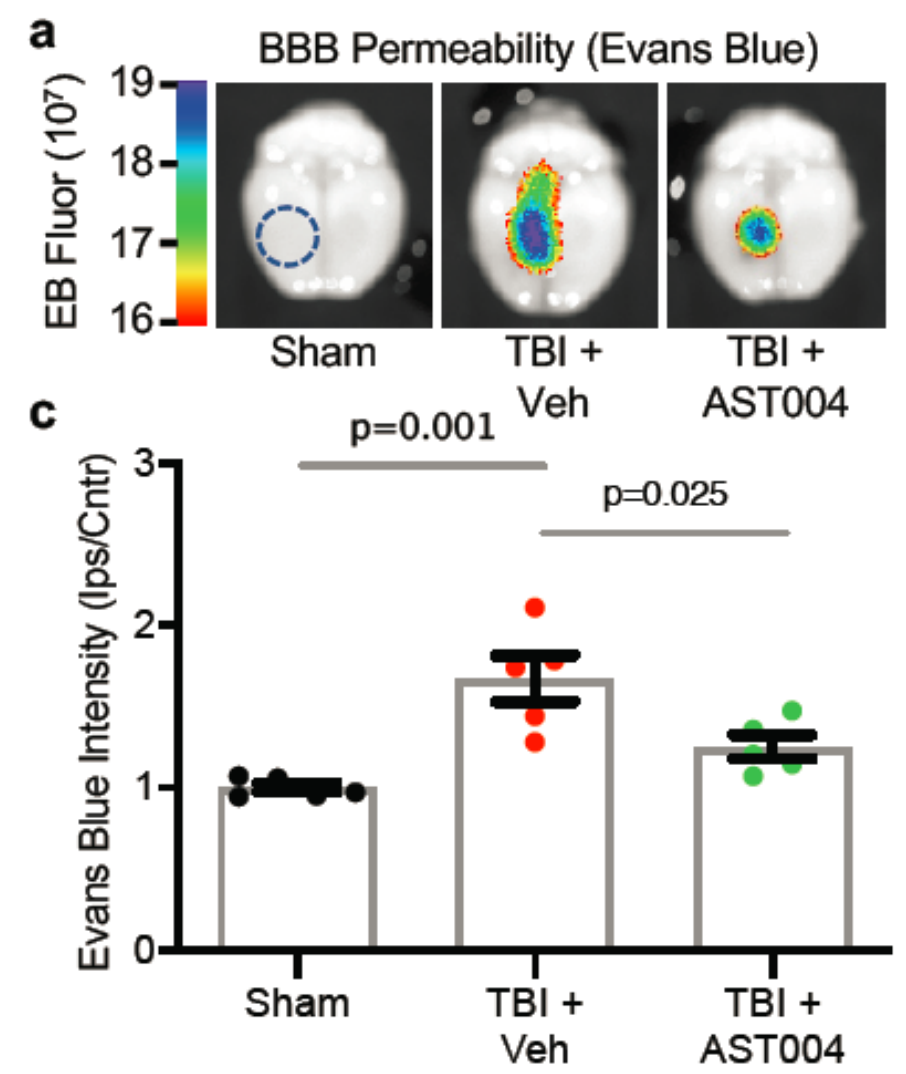

b

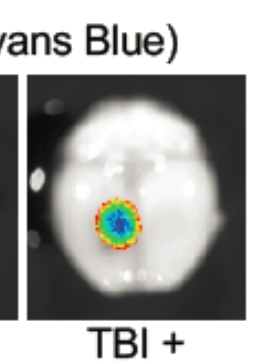

$\mathrm{TBI}+$

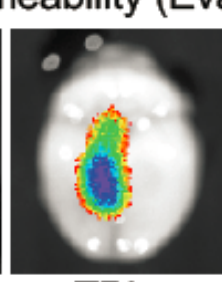

$\mathrm{BI}+$

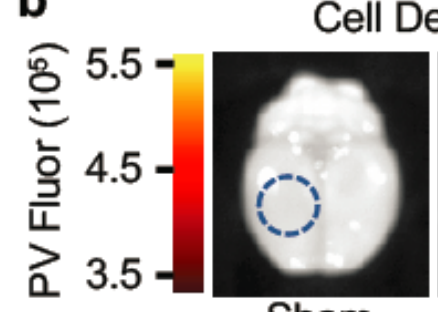

Sham

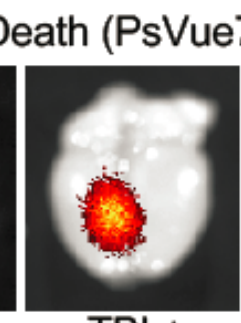

$\mathrm{TBI}+$

d

d

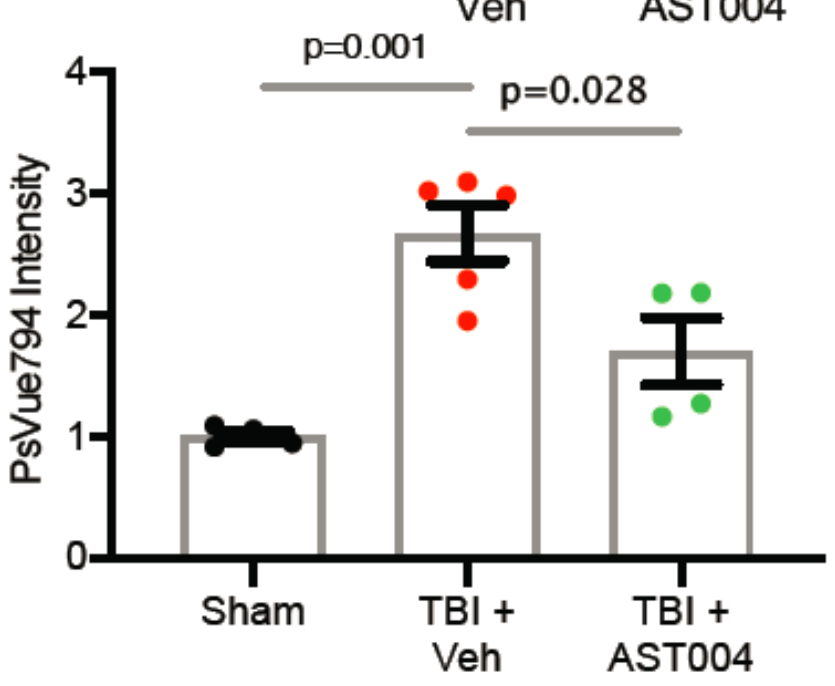

Figure 1

TBI induced increases in BBB Permeability (Evans Blue) and Cell Death (PSVue794) were reduced by AST004 treatment. a, b Images of Evans Blue or PSVue794 injected mice brains 3 days post-TBI, collected on the Xenogen IVIS system. Epi-fluorescence radiance units are the number of photons per second leaving a square centimeter of brain tissue radiating into a solid angle of one steradian (photons $/ \mathrm{sec} / \mathrm{cm} 2 / \mathrm{sr}$ ). c, d Histogram plots of Evans Blue and PSVue794 fluorescence intensity (arbitrary units) normalized to Sham. Size of ROls indicated with blue dashed circles in Sham image panels. $\mathrm{N}=4-5$ mice used per condition as shown by \# of symbols. 

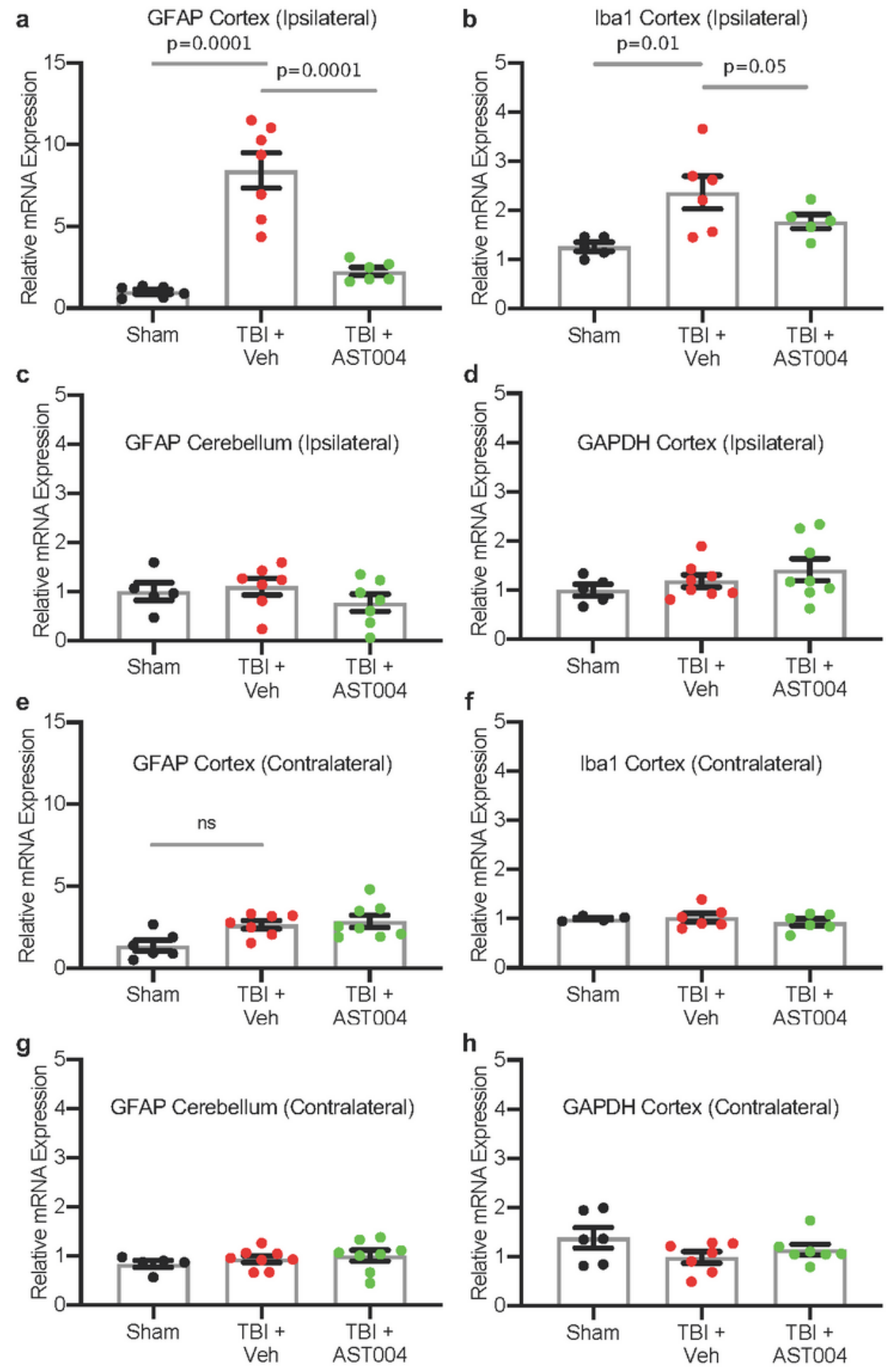

\section{Figure 2}

TBI induced increases in mRNA levels of GFAP and Iba1 in the Cortex are reduced by AST-004 treatments. Mice underwent sham or TBI and received AST-004 treatment $(0.22 \mathrm{mg} / \mathrm{kg})$ or vehicle 30 min postinjury. qRT-PCRs of brain homogenates were performed 7 days post-injury. mRNA levels for GFAP and Iba1 in the Ipsilateral Cortex are shown in histogram plots, as labeled $(a, b)$. GFAP mRNA levels in the ipsilateral cerebellum were not affected by TBI (c).GAPDH mRNA levels in the ipsilateral cortex were also unaffected 
(d). For comparison, contralateral mRNA levels for GFAP, Iba1 and GAPDH in the Cortex and GFAP in the cerebellum are also not significantly different in TBI-induced brain samples (e, $f)$. N = 5-8 mice used per condition as shown by \# of symbols.
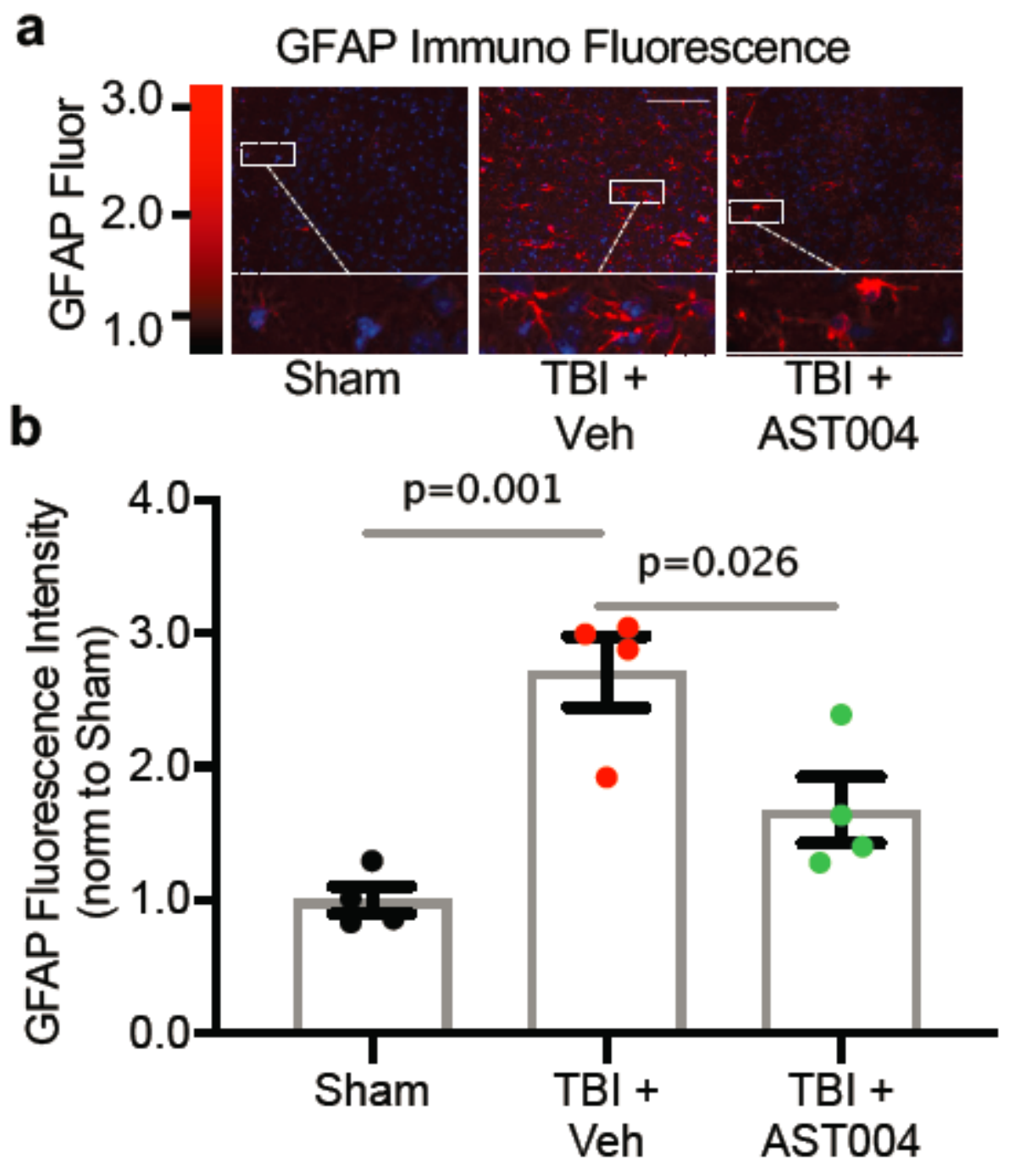

Figure 3

TBI induced increases in GFAP Immunofluorescence are reduced by AST-004 treatments 3 days postinjury. a Image panels of brain sections immunolabeled for GFAP. Mice underwent sham or TBI and received AST-004 treatment $(0.22 \mathrm{mg} / \mathrm{kg})$ or only vehicle 30 min post-injury. Scale bar $100 \mu \mathrm{m} . \mathrm{b}$ Histogram plot of mean fluorescence intensity (arbitrary units) normalized to Sham. $\mathrm{N}=4$ mice used per condition as shown by \# of symbols. 

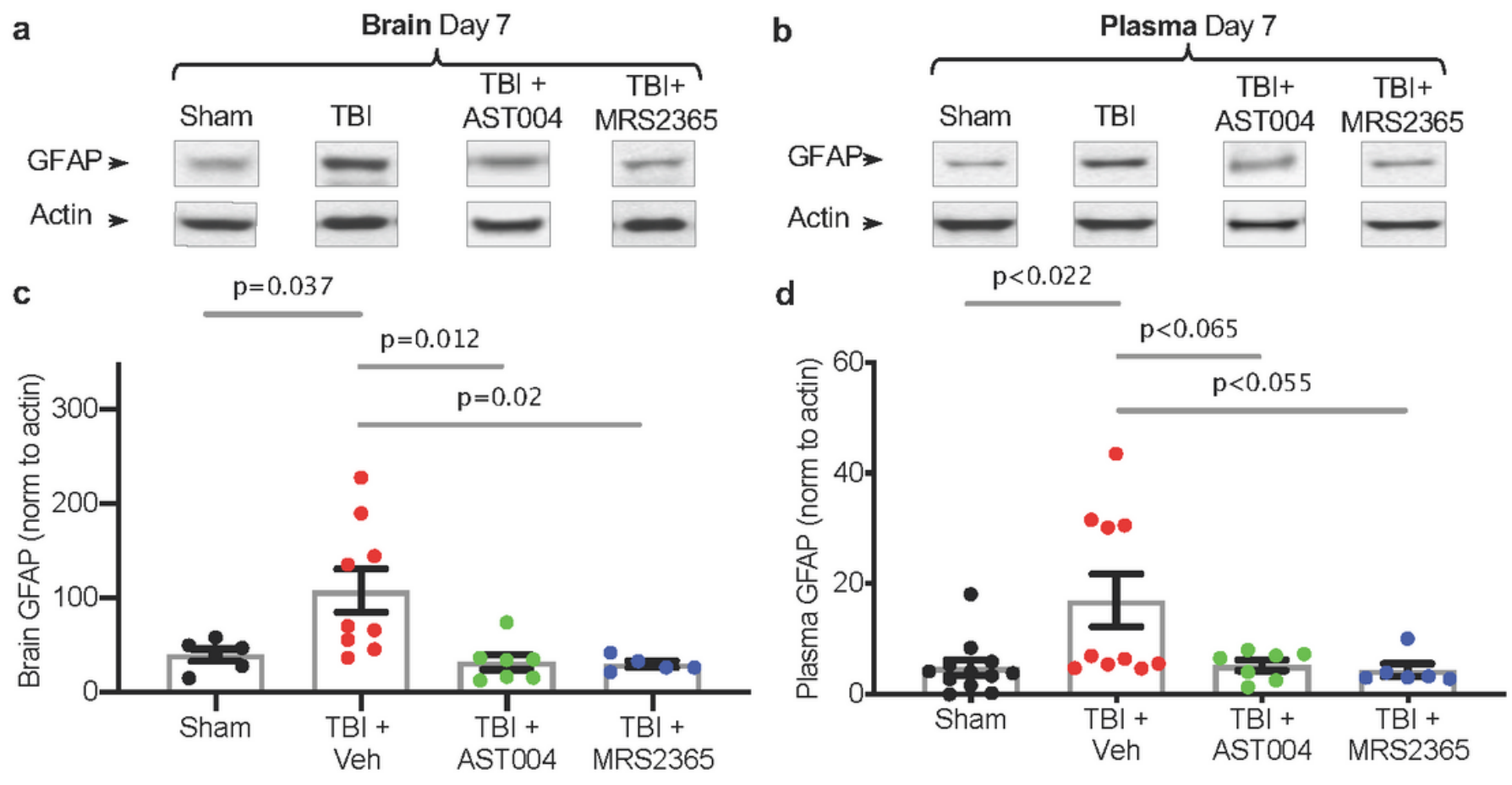

\section{Figure 4}

TBI induced increases in GFAP expression in Brain and Plasma are reduced by AST-004 and MRS2365 treatments 7 days post-injury. Representative Western blots are shown for brain homogenates (a) and plasma (b) collected 7 days post-TBI. (c, d) Histogram plots of GFAP expression levels normalized by actin as labeled. Injured mice or shams were injected with either vehicle, AST-004 $(0.22 \mathrm{mg} / \mathrm{kg})$ or MRS2365 $(0.22 \mathrm{mg} / \mathrm{kg}) 30$ minutes after TBI. $\mathrm{N}=5-11$ mice used per condition as shown by \# of symbols. 

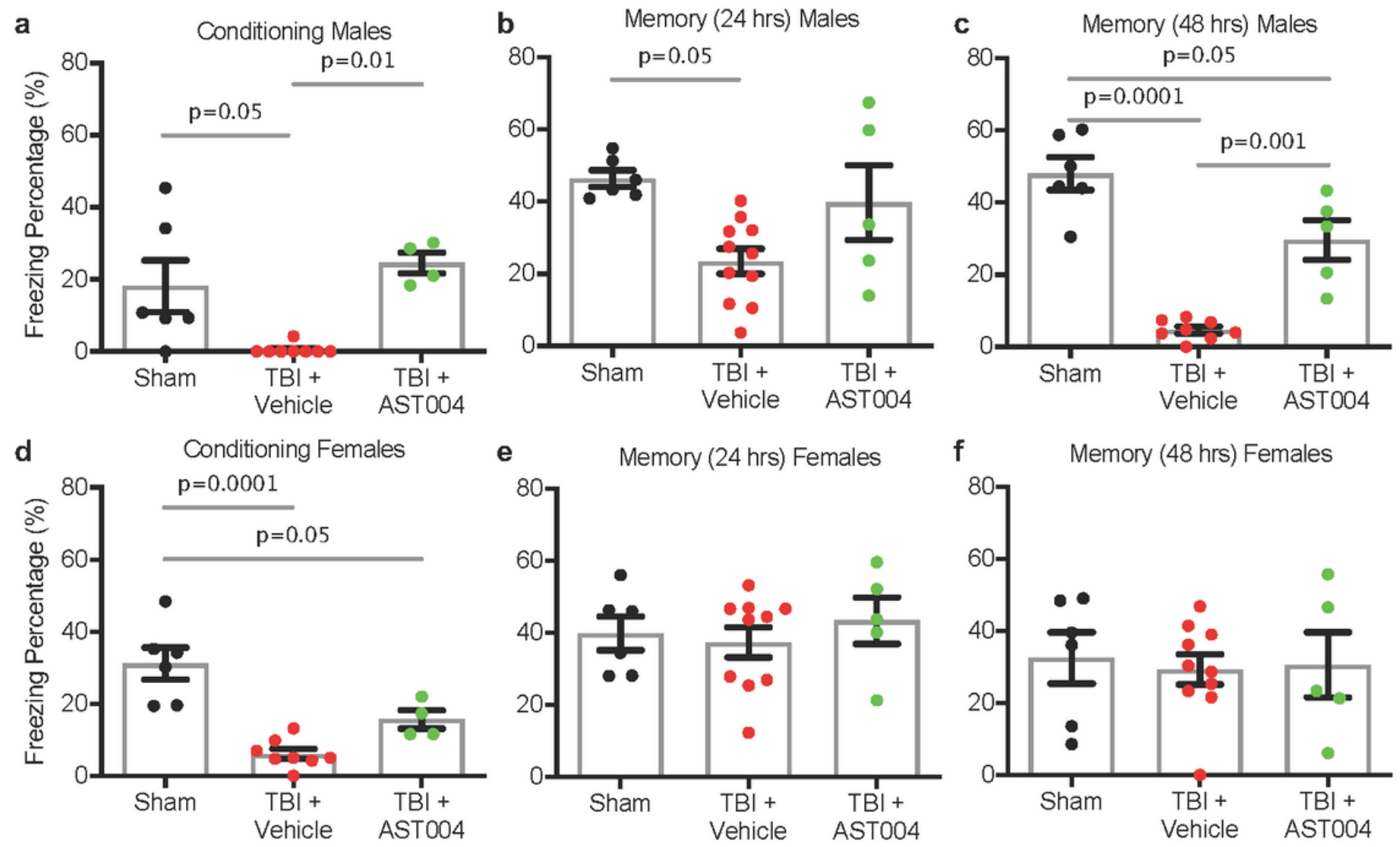

\section{Figure 5}

AST-004 treatments improve spatial memory 30 days post-injury. TBI injured mice or shams were injected with either vehicle or AST-004 $(0.22 \mathrm{mg} / \mathrm{kg}) 30$ minutes after injury. Contextual fear conditioning tests were performed 30 days later. All panels show freezing percentages during contextual fear memory training and testing (24h and $48 \mathrm{~h}$ after TBI). Males (a-c) and females (d-f) were analyzed separately. Note that AST-004 reversed TBI induced impairment in freezing behavior after shock (training session) in both sexes. However, TBI-induced long-memory loss was only observed in male mice, which was significantly improved by AST-004 treatment. $\mathrm{N}=4-11$ mice used per condition as shown by \# of symbols. 

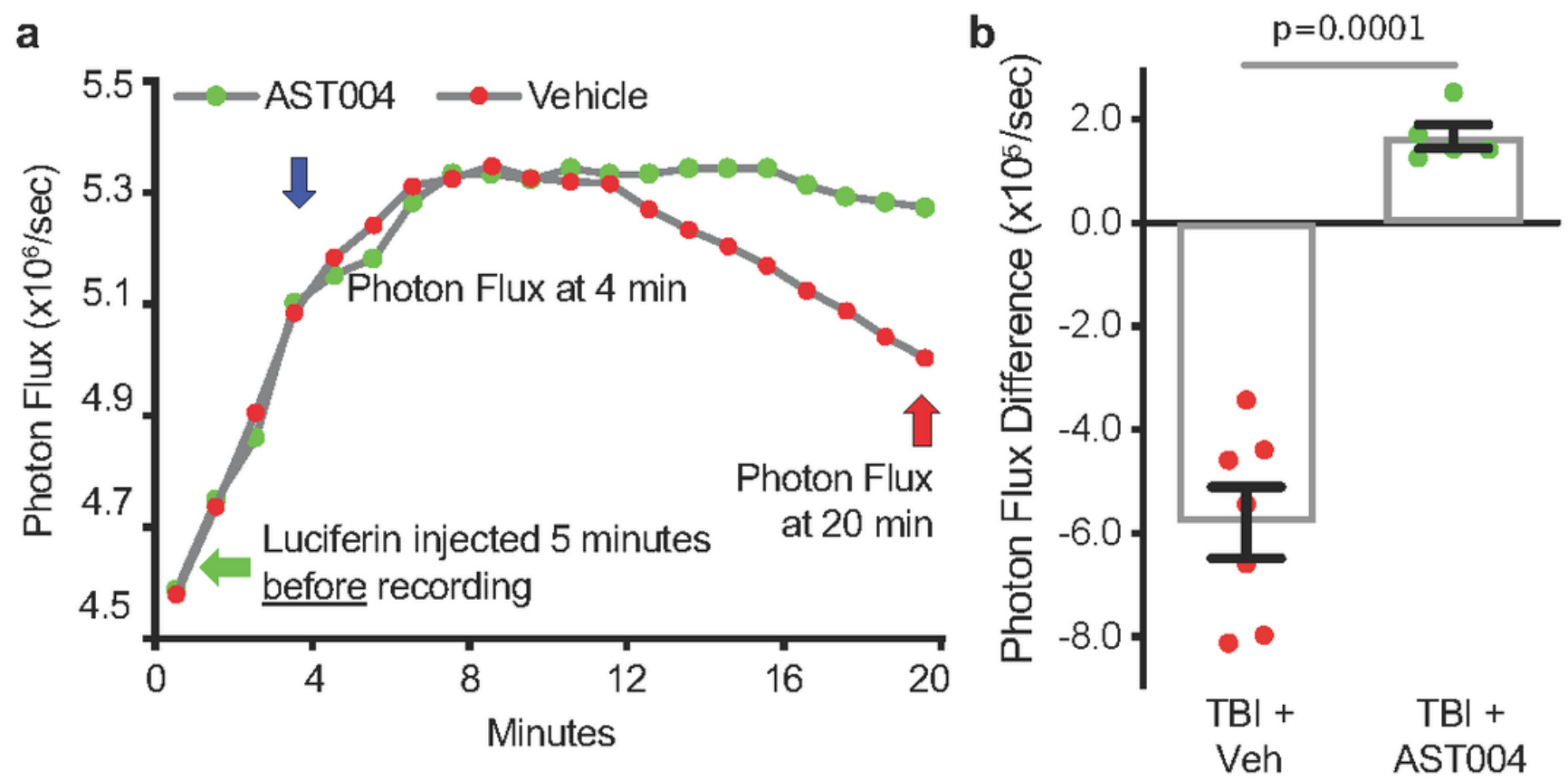

\section{Figure 6}

In vivo bioluminescent imaging indicates AST-004 increases ATP in astrocytes. a Photon flux plots of TBIinjured transgenic mice expressing the Luciferase-reporter gene in astrocytes (GFAP promoter, Jackson Labs) after intraperitoneal injections (IP) of synthetic D-luciferin analogue Cycluc1 (5 mM, $100 \mu \mathrm{l})$. Bioluminescent signals were recorded with a Xenogen IVIS Spectrum Imager, beginning 5 min after Cycluc1 injection (green arrow). Mice were then injected IP with either vehicle or AST-004 at 4 minutes (blue arrow), the photon flux measured at the time of injection and then compared to the photon flux level at 20 minutes (red arrow). Every 24 hours after the initial flux recordings, the same mice were injected again for another photon flux recording, alternating the identity of the test injection between vehicle and AST-004. b Histogram plot showing photon flux differences at 20 minutes compared to corresponding levels at 4 minutes. Note mice injected with AST-004 exhibited positive photon flux difference compared to a loss of photon flux (negative) in vehicle treated mice, consistent with higher ATP-production in astrocytes of AST-004 treated mice. 